\title{
Chemistry and Biology of Deoxy-myo-Inositol Phosphates: Stereospecificity of Substrate Interactions within an Archaeal and a Bacterial IMPase
}

\author{
Adam J. Morgan, Yangling Karen Wang, Mary F. Roberts* and Scott J. Miller ${ }^{\star}$ \\ Department of Chemistry, Merkert Chemistry Center, Boston College, Chestnut Hill, Massachusetts 02467-3860 \\ Email: scott.miller.1@bc.edu
}

\section{Supporting Information}

General Procedures. Proton NMR spectra were recorded on Varian 400 or 300 spectrometers. Proton chemical shifts are reported in ppm ( $\square$ ) relative to internal tetramethylsilane (TMS, $\square 0.0 \mathrm{ppm}$ ) or with the solvent reference relative to TMS employed as the internal standard $\left(\mathrm{CDCl}_{3}, \square 7.26 \mathrm{ppm}\right.$; $\mathrm{d}_{6}-\mathrm{DMSO}, \square 2.50 \mathrm{ppm} ; \mathrm{CD}_{3} \mathrm{OD}$, $\square 3.31 \mathrm{ppm} ; \mathrm{D}_{2} \mathrm{O}, \square 4.79 \mathrm{ppm}$ ). Data are reported as follows: chemical shift (multiplicity [singlet (s), doublet (d), triplet (t), quartet (q), and multiplet $(\mathrm{m})$ ], coupling constants $[\mathrm{Hz}]$, integration). Carbon NMR spectra were recorded on Varian $400(100 \mathrm{MHz})$ or 300 (75 MHz) spectrometers with complete proton decoupling. Carbon chemical shifts are reported in ppm ( $\square$ relative to TMS with the respective solvent resonance as the internal standard $\left(\mathrm{CDCl}_{3}, \square 77.0 \mathrm{ppm}\right)$ except for $\mathrm{D}_{2} \mathrm{O}$ in which case a drop of methanol was added as internal standard $\left(\mathrm{CH}_{3} \mathrm{OH}, \square 49.5 \mathrm{ppm}\right) .{ }^{1}$ Phosphorous NMR spectra were recorded on a Varian $400(162 \mathrm{MHz})$ spectrometer with complete proton decoupling. Phosphorous chemical shifts are reported in ppm $(\square)$ relative to a $85 \% \mathrm{H}_{3} \mathrm{PO}_{4}$ external standard. NMR data were collected at $25^{\circ} \mathrm{C}$, unless otherwise indicated. Infrared spectra were obtained on a Perkin-Elmer Spectrum 1000 spectrometer. Analytical thin-layer chromatography (TLC) was performed using Silica Gel $60 \AA$ F 254 precoated plates $(0.25$ $\mathrm{mm}$ thickness). TLC $\mathrm{R}_{f}$ values are reported. Visualization was accomplished by irradiation with a UV lamp and/or staining with $\mathrm{KMnO}_{4}$ or cerium ammonium molybdenate (CAM) solutions. Flash column chromatography was performed using Silica Gel 60A (32-63 $\square \mathrm{m}){ }^{2}{ }^{2}$ Optical rotations were recorded on a Rudolf Research Analytical Autopol IV Automatic polarimeter at the sodium D line (path length $50 \mathrm{~mm}$ ). High resolution mass spectra were obtained at the Mass Spectrometry Facility of either Boston College (Chestnut Hill, MA) or the University of Illinois (Urbana-Champaign, IL). The method of ionization is given in parentheses.

Analytical and preparative reverse phase and normal phase HPLC were performed on a Rainin SD-200 chromatograph equipped with a single wavelength UV detector (214 $\mathrm{nm}$ or $254 \mathrm{~nm}$ ). Analytical normal phase HPLC was performed on a Hewlett-Packard 1100 Series chromatograph equipped with a diode array detector $(214 \mathrm{~nm}$ and $254 \mathrm{~nm})$. All reactions were carried out under an argon or nitrogen atmosphere employing ovenand flame-dried glassware. All solvents were distilled from appropriate drying agents prior to use. Diphenyl chlorophosphate was distilled prior to use and stored in a Schlenk tube for no more than 2 weeks. 2,4,6-Tri-O-benzyl-myo-inositol-monophosphates (5+ ent-5) were prepared according to previously published methodology. ${ }^{3}$ The structures of fully deprotected biscyclohexylamine salts $\mathbf{8}$, ent-8, 11, and ent-11 were confirmed by gDQCOSY $^{4}$ and P-H HETCOR ${ }^{5}$ experiments. 
<smiles>O=P(OCc1ccccc1)(Oc1ccccc1)OC1[C@H](O)[C@H](O)C(OC(=S)Oc2ccccc2)[C@H](O)[C@H]1Cc1ccccc1</smiles>

\section{2,4,6-Tri-O-benzyl-D-myo-inositol-1-diphenylphosphate-3- O-thiocarbonic acid phenyl ester (6).}

To a stirred solution of $5(0.300 \mathrm{~g}, 0.440 \mathrm{mmol})$ in dichloromethane $(10.0 \mathrm{~mL})$ was added pyridine $(214 \mu \mathrm{L}, 2.64$ mmol) followed by $N, N$-dimethylamino pyridine $(16.0 \mathrm{mg}$, $0.132 \mathrm{mmol})$. Phenyl chlorothionoformate $(304 \mu \mathrm{L}, 2.20$ mmol) was then added and the reaction stirred for 2.5 hours at which time methanol $(2.00$ $\mathrm{mL}$ ) was added and the reaction was concentrated under reduced pressure to afford a yellow oily solid. Thiocarbonate (6) was purified using silica gel flash chromatography eluting with a gradient of $10-20 \%$ ethyl acetate/hexanes to afford pure product as a clear oil (253 mg, 70\% yield). ${ }^{1} \mathbf{H}$ NMR $\left(\mathrm{CDCl}_{3}, 400 \mathrm{MHz}\right) \square 7.41-7.11$ (m, 28H); 7.00-6.95 $(\mathrm{m}, 2 \mathrm{H}) ; 5.32(\mathrm{dd}, \mathrm{J}=2.0,10.4 \mathrm{~Hz}, 1 \mathrm{H}) ; 4.82-4.64(\mathrm{~m}, 8 \mathrm{H}) ; 4.12(\mathrm{t}, \mathrm{J}=10.0 \mathrm{~Hz}, 1 \mathrm{H})$; $4.02(\mathrm{t}, \mathrm{J}=9.6 \mathrm{~Hz}, 1 \mathrm{H}) ; 3.68(\mathrm{dt}, \mathrm{J}=2.4,9.2 \mathrm{~Hz}, 1 \mathrm{H}) ; 2.51(\mathrm{~d}, \mathrm{~J}=2.8 \mathrm{~Hz}, 1 \mathrm{H}) ;{ }^{13} \mathbf{C ~ N M R}$ $\left(\mathrm{CDCl}_{3}, 100 \mathrm{MHz}\right) \square 193.8,153.0,138.0,137.7,129.7,129.6,129.4,129.3,128.2,127.8$, $127.7,127.6,127.5,126.6,125.3,121.6,120.0,119.9,119.8,82.7,79.5,79.4,78.9,78.7$, 75.9, 75.4, 75.3, 75.2, 74.4; ${ }^{31} \mathbf{P}$ NMR $\left(\mathrm{CDCl}_{3}, 162 \mathrm{MHz}\right) \square-11.6$; IR (film, cm $\left.{ }^{-1}\right) 3427$, 2357, 1640, 1590, 1489, 1451, 1287, 1187; TLC $\mathbf{R}_{\boldsymbol{f}} 0.34$ (30\% ethyl acetate/hexanes); Exact mass calcd for $\left[\mathrm{C}_{46} \mathrm{H}_{43} \mathrm{O}_{10} \mathrm{PS}+\mathrm{Na}\right]+$ requires $\mathrm{m} / z$ 841.2212. Found 841.2249 $(\mathrm{ESI}+) ;[\square]_{\mathbf{D}}=+31.0\left(1.0, \mathrm{CH}_{2} \mathrm{Cl}_{2}\right)$.<smiles>CC(=O)O[C@@H]1[C@H](OCc2ccccc2)[C@@H](OP(=O)(c2ccccc2)c2ccccc2)[C@@H](Br)[C@@H](OC(=S)Oc2ccccc2)[C@@H]1OCc1ccccc1</smiles>

5-O-Acetate-2,4,6-tri-O-benzyl-D-myo-inositol-1diphenylphosphate-3-O-thiocarbonic acid phenyl ester. To a stirred solution of $6(0.230 \mathrm{~g}, 0.281 \mathrm{mmol})$ in dichloromethane $(5.00 \mathrm{~mL})$ was added triethylamine $(90.0$ $\mu \mathrm{L}, 0.646 \mathrm{mmol}$ ) followed by $N, N$-dimethylamino pyridine $(10.0 \mathrm{mg}, 0.0840 \mathrm{mmol})$. Acetic anhydride $(53.0 \mu \mathrm{L}, 0.562$ mmol) was then added and the reaction stirred for 4 hours at which time the reaction was diluted with dichloromethane $(20.0 \mathrm{~mL})$ and washed with water $(3 \times 20 \mathrm{~mL})$. The organic layer was then dried $\left(\mathrm{MgSO}_{4}\right)$, filtered, and concentrated under reduced pressure to afford pure monoacetate as a clear oil $(220 \mathrm{mg}, 91 \%$ Yield $) .{ }^{1} \mathbf{H} \mathbf{~ N M R}\left(\mathrm{CDCl}_{3}, 400\right.$ $\mathrm{MHz}) \square 7.41-7.10(\mathrm{~m}, 28 \mathrm{H}) ; 7.00-6.94(\mathrm{~m}, 2 \mathrm{H}) ; 5.39(\mathrm{dd}, \mathrm{J}=2.0,10.4 \mathrm{~Hz}, 1 \mathrm{H}) ; 5.24$ (t, J $=9.6 \mathrm{~Hz}, 1 \mathrm{H}) ; 4.78-4.64(\mathrm{~m}, 6 \mathrm{H}) ; 4.62-4.50(\mathrm{~m}, 2 \mathrm{H}) ; 4.21(\mathrm{t}, \mathrm{J}=10.0 \mathrm{~Hz}, 1 \mathrm{H}) ; 4.11(\mathrm{t}, \mathrm{J}$ $=9.6 \mathrm{~Hz}, 1 \mathrm{H}) ; 1.80(\mathrm{~s}, 3 \mathrm{H}) ;{ }^{13} \mathbf{C ~ N M R}\left(\mathrm{CDCl}_{3}, 100 \mathrm{MHz}\right) \square 193.5,169.2,152.9,150.1$, $137.7,137.6,129.6,129.4,128.2$, 128.1 127.7, 127.6, 127.5, 127.3, 126.6, 125.3, 121.6, $120.7,119.9,119.8,119.7,82.5,78.9,78.8,77.8,77.7,76.7,75.5,75.0,74.9,72.8,20.8$; ${ }^{31} \mathbf{P}$ NMR $\left(\mathrm{CDCl}_{3}, 162 \mathrm{MHz}\right) \square-12.0$; IR (film, $\left.\mathrm{cm}^{-1}\right)$ 3065, 3023, 2925, 2351, 1746, 1587, 1483, 1453, 1361, 1300; TLC $\mathbf{R}_{\boldsymbol{f}} 0.20$ (20\% ethyl acetate/hexanes); Exact mass

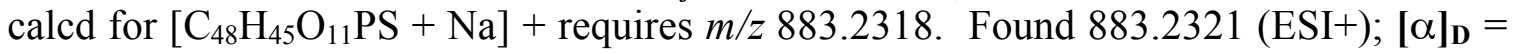
$+20.8\left(1.0, \mathrm{CH}_{2} \mathrm{Cl}_{2}\right)$. 


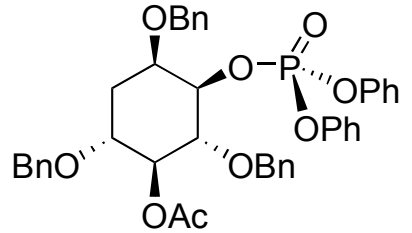

\section{5-O-Acetate-2,4,6-tri-O-benzyl-D-myo-inositol-3-deoxy- 1-diphenyl phosphate (7).}

To a stirred solution of 5-O-acetate-2,4,6-tri-O-benzyl-D-myoinositol-1-diphenylphosphate-3-O-thiocarbonic acid phenyl ester $(0.270 \mathrm{~g}, 0.314 \mathrm{mmol})$ in toluene $(10.0 \mathrm{~mL})$ was added tributyltin hydride $(253 \mu \mathrm{L}, 0.941 \mathrm{mmol})$ followed by AIBN (15.0 mg, $0.0940 \mathrm{mmol})$. The reaction was stirred at reflux and monitored by thin layer chromatography. Starting material was consumed after 2 hours and the reaction was cooled to room temperature and concentrated under reduced pressure to afford a clear oil. Deoxy-inositol 7 was purified using silica gel flash chromatography eluting with a gradient of $15-20 \%$ ethyl acetate/hexanes to afford pure product as a clear oil (155 mg, 70\% yield). ${ }^{1} \mathbf{H}$ NMR $\left(\mathrm{CDCl}_{3}, 400 \mathrm{MHz}\right) \square 7.40-7.05(\mathrm{~m}, 25 \mathrm{H}) ; 5.14(\mathrm{t}, \mathrm{J}=9.6 \mathrm{~Hz}, 1 \mathrm{H}) ; 4.70(\mathrm{~d}, \mathrm{~J}=11.6 \mathrm{~Hz}$, $1 \mathrm{H}) ; 4.61$ (ddd, J = 3.6, 8.2, 8.6 Hz, 6H); 4.52 (d, J = $11.2 \mathrm{~Hz}, 1 \mathrm{H}) ; 4.50-4.34(\mathrm{~m}, 4 \mathrm{H})$; $4.15(\mathrm{~s}, 1 \mathrm{H}) ; 3.99(\mathrm{t}, \mathrm{J}=9.6 \mathrm{~Hz}, 1 \mathrm{H}) ; 3.76(\mathrm{ddd}, \mathrm{J}=4.4,9.6,11.4 \mathrm{~Hz}, 1 \mathrm{H}) ; 2.23$ (dt, J = 4.4, $14.4 \mathrm{~Hz}, 1 \mathrm{H}) ; 1.89(\mathrm{~s}, 3 \mathrm{H}) ; 1.41(\mathrm{dt}, \mathrm{J}=2.0,13.0 \mathrm{~Hz}, 1 \mathrm{H}) ;{ }^{13} \mathbf{C}$ NMR $\left(\mathrm{CDCl}_{3}, 100\right.$ $\mathrm{MHz}) \square 138.1,129.6,128.2,128.0,127.7,127.6,127.5,127.4,127.2,125.2,125.1$, $120.1,120.0,119.9,119.8,81.7,78.2,76.1,75.8,75.0,74.5,73.9,72.4,71.9,36.4,30.3$, 21.0; ${ }^{31} \mathbf{P}$ NMR $\left(\mathrm{CDCl}_{3}, 162 \mathrm{MHz}\right) \square-11.5$; IR (film, $\left.\mathrm{cm}^{-1}\right)$ 3062, 3031, 2924, 2357, 2326, 1741, 1590, 1489, 1451, 1287, 1237, 1193; TLC $\mathbf{R}_{f} 0.15$ (30\% ethyl acetate/hexanes); Exact mass calcd for $\left[\mathrm{C}_{41} \mathrm{H}_{41} \mathrm{O}_{9} \mathrm{P}+\mathrm{Na}\right]+$ requires $\mathrm{m} / z$ 731.2386. Found $731.2391(\mathrm{ESI}+) ;[\square]_{\mathbf{D}}=-23.0\left(1.0, \mathrm{CH}_{2} \mathrm{Cl}_{2}\right)$.<smiles>O=P([O-])([O-])O[C@H]1[C@@H](O)C[C@@H](O)[C@H](O)[C@H]1O</smiles>

3-Deoxy-D-myo-inositol-1-phosphate biscyclohexylamine salt (8). Ammonia $(7 \mathrm{~mL})$ was condensed into $\mathrm{THF}(2 \mathrm{~mL})$ at $-78{ }^{\circ} \mathrm{C}$ under an atmosphere of argon. (Caution: it is important to use argon and not nitrogen when working with lithium.) Lithium wire $(0.5 \mathrm{~cm} \times 3.2 \mathrm{~mm}$ diameter) was delivered causing the solution to turn a deep blue color. A solution of $7(0.062 \mathrm{~g}, 0.087 \mathrm{mmol})$ in THF $(1 \mathrm{~mL})$ was then added dropwise and the reaction continued to stir for $15 \mathrm{~min}$ at which time the solution became white. A second piece of lithium wire $(0.5 \mathrm{~cm} \times 3.2 \mathrm{~mm}$ diameter $)$ was then added and the reaction stirred for $30 \mathrm{~min}$, upon which time it was quenched with small pieces of ice. The solution was slowly warmed to room temperature and the ammonia was evaporated under a stream of argon. The resulting solids were taken up in $2 \mathrm{~mL}$ of $\mathrm{H}_{2} \mathrm{O}$ and passed through a column of Dowex 500WX2-200 ion exchange resin eluting with $\mathrm{H}_{2} \mathrm{O}$. The acidic fractions were collected and stirred with $1.2 \mathrm{~mL}$ of cyclohexylamine for 1 hour. The $\mathrm{H}_{2} \mathrm{O}$ and excess amine were removed by lyophilization to yield $\mathbf{8}$ (39 mg, 99\% yield) as an off-white solid. ${ }^{1} \mathbf{H}$ NMR $\left(\mathrm{D}_{2} \mathrm{O}, 400 \mathrm{MHz}\right) \square 4.21(\mathrm{~d}$, $\mathrm{J}=3.2 \mathrm{~Hz}, 1 \mathrm{H}) ; 3.87(\mathrm{dt}, \mathrm{J}=2.8,9.2 \mathrm{~Hz}, 1 \mathrm{H}) ; 3.76(\mathrm{dt}, \mathrm{J}=4.4,9.3 \mathrm{~Hz}, 1 \mathrm{H}) ; 3.69(\mathrm{t}, \mathrm{J}=$ $9.2 \mathrm{~Hz}, 1 \mathrm{H}) ; 3.31(\mathrm{t}, \mathrm{J}=8.8 \mathrm{~Hz}, 1 \mathrm{H}) ; 3.18-3.04(\mathrm{~m}, 2 \mathrm{H}) ; 2.10(\mathrm{dt}, \mathrm{J}=4.4,14.4 \mathrm{~Hz}, 1 \mathrm{H})$; 1.95 (br. s, 4H); 1.78 (br. s, 4H); 1.68-1.54 (m, 3H); 1.38-1.24 (m, 8H); 1.22-1.08 (m, $2 \mathrm{H}) ;{ }^{13} \mathbf{C}$ NMR $\left(\mathrm{D}_{2} \mathrm{O}, 100 \mathrm{MHz}\right) \square 77.9,77.3,73.0,68.7,68.5,51.0,35.1,31.0,25.0$, $24.5 ;{ }^{31} \mathbf{P}$ NMR $\left(\mathrm{D}_{2} \mathrm{O}, 162 \mathrm{MHz}\right) \square 5.0$; Exact mass calcd for $\left[\mathrm{C}_{6} \mathrm{H}_{13} \mathrm{O}_{8} \mathrm{P}+\mathrm{C}_{6} \mathrm{H}_{14} \mathrm{~N}\right]+$ requires $m / z$ 344.147431. Found 344.147600 (FAB); [ $]_{\mathbf{D}}=-8.6\left(1.0, \mathrm{H}_{2} \mathrm{O}\right.$ at $\mathrm{pH}$ 9). 
2-D NMR experiments for 3-deoxy-D-myo-inositol-1phosphate biscyclohexylamine salt (8).
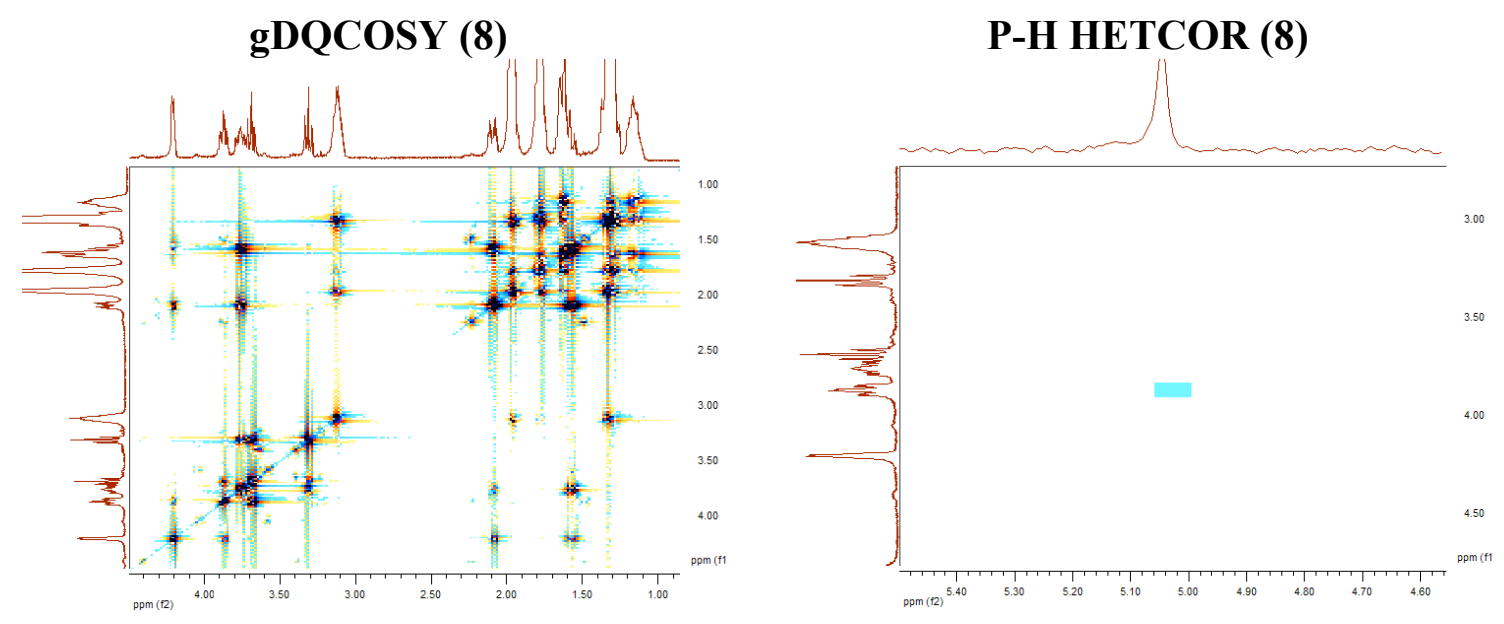

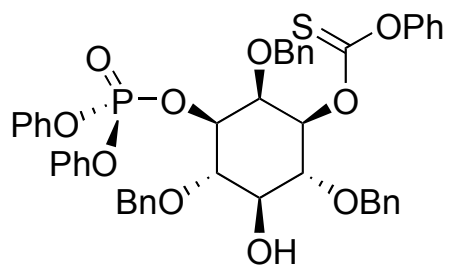

2,4,6-Tri-O-benzyl-D-myo-inositol-3-diphenylphosphate1-O-thiocarbonic acid phenyl ester (ent-6). Spectral data matches that reported for 6 . [ $[\square]_{\mathbf{D}}=-30.6\left(1.0, \mathrm{CH}_{2} \mathrm{Cl}_{2}\right)$.

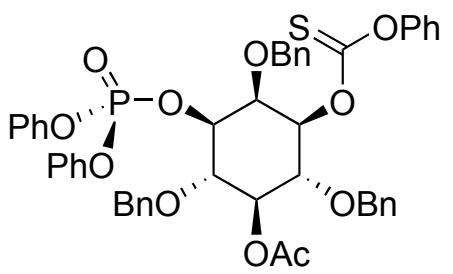

5-O-Acetate-2,4,6-Tri-O-benzyl-D-myo-inositol-3diphenylphosphate-1-O-thiocarbonic acid phenyl ester. Spectral data matches that reported for 5-O-acetate-2,4,6tri-O-benzyl-D-myo-inositol-1-diphenylphosphate-3-Othiocarbonic acid phenyl ester. $\left[\left.\square\right|_{\mathbf{D}}=-21.2\left(1.0, \mathrm{CH}_{2} \mathrm{Cl}_{2}\right)\right.$.

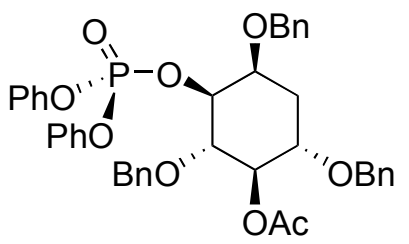

5-O-Acetate-2,4,6-tri-O-benzyl-D-myo-inositol-1-deoxy3-diphenyl phosphate (ent-7). Spectral data matches that reported for 7 . $[\square]_{\mathbf{D}}=+23.6\left(1.0, \mathrm{CH}_{2} \mathrm{Cl}_{2}\right)$. 
<smiles>CO[P+](=O)(O)O[C@H]1[C@@H](O)C[C@@H](O)[C@H](O)[C@H]1O</smiles>

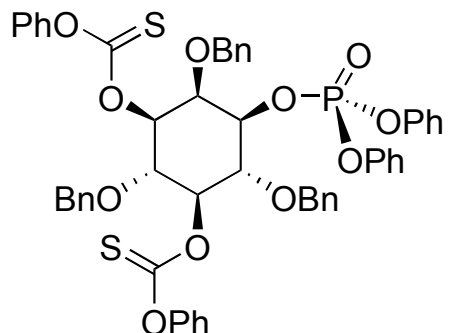

1-Deoxy-D-myo-inositol-3-phosphate biscyclohexylamine

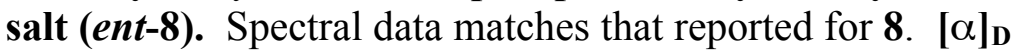
$=+7.8\left(1.0, \mathrm{H}_{2} \mathrm{O}\right.$ at $\left.\mathrm{pH} 9\right)$.

time methanol $(2.00 \mathrm{~mL})$ was added and the reaction was concentrated under reduced pressure to afford a yellow oily solid. Dithiocarbonate (9) was purified using silica gel flash chromatography eluting with a gradient of $10-20 \%$ ethyl acetate/hexanes to afford pure product as a clear oil $\left(439 \mathrm{mg}, 70 \%\right.$ Yield). ${ }^{1} \mathbf{H}$ NMR $\left(\mathrm{CDCl}_{3}, 400 \mathrm{MHz}\right) \square 7.45-$ $7.05(\mathrm{~m}, 31 \mathrm{H}) ; 6.97-6.93(\mathrm{~m}, 2 \mathrm{H}) ; 6.77-6.72(\mathrm{~m}, 2 \mathrm{H}) ; 5.83(\mathrm{t}, \mathrm{J}=9.5 \mathrm{~Hz}, 1 \mathrm{H}) ; 5.40(\mathrm{dd}, \mathrm{J}$ $=2.3,10.2 \mathrm{~Hz}, 1 \mathrm{H}) ; 4.82-4.68(\mathrm{~m}, 6 \mathrm{H}) ; 4.66(\mathrm{~s}, 2 \mathrm{H}) ; 4.42(\mathrm{t}, \mathrm{J}=9.8 \mathrm{~Hz}, 1 \mathrm{H}) ; 4.32(\mathrm{t}, \mathrm{J}=$ $9.6 \mathrm{~Hz}, 1 \mathrm{H}) ;{ }^{13} \mathrm{C} \mathrm{NMR}\left(\mathrm{CDCl}_{3}, 100 \mathrm{MHz}\right) \square 194.1,193.5,153.2,153.0,150.2,150.1$, $137.5,129.7,129.6,129.4,129.3,129.2,128.2$, 128.0, 127.7, 127.6, 127.5, 127.4, 126.6, $126.4,125.9,125.3,121.6,120.7,119.9,119.8,83.3,82.3,82.2,78.5,77.7,76.7,75.6$,

75.4, 75.3, 75.2; ${ }^{31} \mathbf{P}$ NMR $\left(\mathrm{CDCl}_{3}, 162 \mathrm{MHz}\right) \square-11.8$; TLC $\mathbf{R}_{\boldsymbol{f}} 0.38$ (30\% ethyl acetate/hexanes); Exact mass calcd for $\left[\mathrm{C}_{53} \mathrm{H}_{47} \mathrm{O}_{11} \mathrm{P}+\mathrm{Na}\right]+$ requires $\mathrm{m} / z$ 977.2195. Found 977.2202 (ESI+); [D] $]_{\mathbf{D}}=+4.8\left(1.0, \mathrm{CH}_{2} \mathrm{Cl}_{2}\right)$.<smiles>O=P(OC1[C@@H](O)C[C@@H](O)C[C@@H]1OP(=O)(c1ccccc1)c1ccccc1)(c1ccccc1)c1ccccc1</smiles>

2,4,6-Tri-O-benzyl-D-myo-inositol-3,5-dideoxy-1-diphenyl phosphate (10). Dithiocarbonate 9 (439 $\mathrm{mg}, 0.460 \mathrm{mmol})$ was dissolved in toluene $(20 \mathrm{~mL})$ and tributyltin hydride $(370 \mu \mathrm{L}$, $1.38 \mathrm{mmol})$ was added follwed by AIBN (15.0 mg, 0.0920 $\mathrm{mmol})$. The reaction was then heated to reflux and continued to stir at reflux for 2 hours. The reaction was then cooled to room temperature and concentrated under reduced pressure to afford a clear oil. Dideoxy (10) was purified via column chromatography eluting with $20 \%$ ethyl acetate/hexanes to afford pure product as a clear oil (182 mg, 60\% Yield) ${ }^{1} \mathbf{H}$ NMR $\left(\mathrm{CDCl}_{3}, 400 \mathrm{MHz}\right) \square 7.40-7.08(\mathrm{~m}, 25 \mathrm{H}) ; 4.64$ $(\mathrm{dt}, \mathrm{J}=2.8,8.8 \mathrm{~Hz}, 2 \mathrm{H}) ; 4.57(\mathrm{~s}, 2 \mathrm{H}) ; 4.52-4.38(\mathrm{~m}, 4 \mathrm{H}) ; 4.16-4.10(\mathrm{~m}, 1 \mathrm{H}) ; 3.94(\mathrm{dt}, \mathrm{J}=$ $4.8,10 \mathrm{~Hz}, 1 \mathrm{H}) ; 3.80-3.71(\mathrm{~m}, 1 \mathrm{H}) ; 2.48-2.38(\mathrm{~m}, 1 \mathrm{H}) ; 2.32-2.23(\mathrm{~m}, 1 \mathrm{H}) ; 1.56-1.42(\mathrm{~m}$, $2 \mathrm{H}) ;{ }^{13} \mathrm{C}$ NMR $\left(\mathrm{CDCl}_{3}, 100 \mathrm{MHz}\right) \square 138.3,138.2,129.5,129.4,128.3,128.1,128.0$, $127.5,127.4,127.3,125.1,125.0,120.1,120.0,119.9,75.2,74.5,74.4,72.2,72.0,71.1$, 70.7, 35.6, 34.0; ${ }^{31} \mathbf{P}$ NMR $\left(\mathrm{CDCl}_{3}, 162 \mathrm{MHz}\right) \square-11.3$; IR (film, $\left.\mathrm{cm}^{-1}\right)$ 3068, 3031, 2937, $2867,2357,2332,1772,1590,1501,1457,1363,1294,1193$; TLC $\mathbf{R}_{\boldsymbol{f}} 0.22$ (30\% ethyl 
acetate/hexanes); Exact mass calcd for $\left[\mathrm{C}_{39} \mathrm{H}_{39} \mathrm{O}_{7} \mathrm{P}+\mathrm{Na}\right]+$ requires $\mathrm{m} / z \quad 673.2331$. Found $673.2335(\mathrm{ESI}+) ;[\square]_{\mathbf{D}}=-21.6\left(1.0, \mathrm{CH}_{2} \mathrm{Cl}_{2}\right)$.<smiles>O=P([O-])([O-])O[C@H]1[C@@H](O)C[C@@H](O)C[C@H]1O</smiles>
3,5-Dideoxy-D-myo-inositol-1-phosphate biscyclohexylamine salt (11). Ammonia (7 mL) was condensed into THF $(2 \mathrm{~mL})$ at -78 ${ }^{\circ} \mathrm{C}$ under an atmosphere of argon. (Caution: it is important to use argon and not nitrogen when working with lithium.) Lithium wire ( $0.5 \mathrm{~cm} \times 3.2 \mathrm{~mm}$ diameter) was delivered causing the solution to turn a deep blue color. A solution of $10(0.086 \mathrm{~g}, 0.132 \mathrm{mmol})$ in THF $(1 \mathrm{~mL})$ was then added dropwise and the reaction continued to stir for $15 \mathrm{~min}$ at which time the solution became white. A second piece of lithium wire $(0.5 \mathrm{~cm} \times 3.2 \mathrm{~mm}$ diameter $)$ was then added and the reaction stirred for $30 \mathrm{~min}$, upon which time it was quenched with small pieces of ice. The solution was slowly warmed to room temperature and the ammonia was evaporated under a stream of argon. The resulting solids were taken up in $2 \mathrm{~mL}$ of $\mathrm{H}_{2} \mathrm{O}$ and passed through a column of Dowex 500WX2-200 ion exchange resin eluting with $\mathrm{H}_{2} \mathrm{O}$. The acidic fractions were collected and stirred with $1.2 \mathrm{~mL}$ of cyclohexylamine for 1 hour. The $\mathrm{H}_{2} \mathrm{O}$ and excess amine were removed by lyophilization to yield 11 (57 mg, 99\% yield) as an off-white solid. ${ }^{1} \mathbf{H}$ NMR $\left(\mathrm{D}_{2} \mathrm{O}, 500 \mathrm{MHz}\right) \square 4.27$ (br d, J = 3.0 Hz, 1H); 4.08-4.00 (m, 1H); 3.93 $(\mathrm{dt}, \mathrm{J}=6.5,9.0 \mathrm{~Hz}, 1 \mathrm{H}) ; 3.86(\mathrm{dt}, \mathrm{J}=2.5,8.5 \mathrm{~Hz}, 1 \mathrm{H}) ; 3.11(\mathrm{~m}, 2 \mathrm{H}) ; 2.27(\mathrm{dd}, \mathrm{J}=3.0$, $12.0 \mathrm{~Hz}, 1 \mathrm{H}) ; 2.15(\mathrm{dd}, \mathrm{J}=3.0,13.5 \mathrm{~Hz}, 1 \mathrm{H}) ; 1.99$ (br. s, $4 \mathrm{H}) ; 1.81$ (br. s, $4 \mathrm{H}) ; 1.71-1.55$ $(\mathrm{m}, 3 \mathrm{H}) ; 1.50-1.30(\mathrm{~m}, 9 \mathrm{H}) ; 1.29-1.10(\mathrm{~m}, 2 \mathrm{H}) ;{ }^{13} \mathbf{C} \mathbf{N M R}\left(\mathrm{D}_{2} \mathrm{O}, 100 \mathrm{MHz}\right) \square 79.0,69.3$, 68.4, 64.5, 51.0, 40.6, 38.7, 31.1, 25.0, 24.5; ${ }^{31} \mathbf{P}$ NMR $\left(\mathrm{D}_{2} \mathrm{O}, 162 \mathrm{MHz}\right) \square 5.1$ Exact mass calcd for $\left[\mathrm{C}_{6} \mathrm{H}_{12} \mathrm{O}_{7} \mathrm{P}+\mathrm{Na}\right]+$ requires $m / z$ 227.0321. Found $227.0322(\mathrm{ESI}+)$; [ $\left[\mathrm{l}_{\mathbf{D}}\right.$ $=-10.2\left(1.0, \mathrm{H}_{2} \mathrm{O}\right.$ at $\left.\mathrm{pH} 9\right)$.<smiles>O=P([O-])([O-])OC1[C@@H](O)C[C@@H](O)C[C@H]1O</smiles>

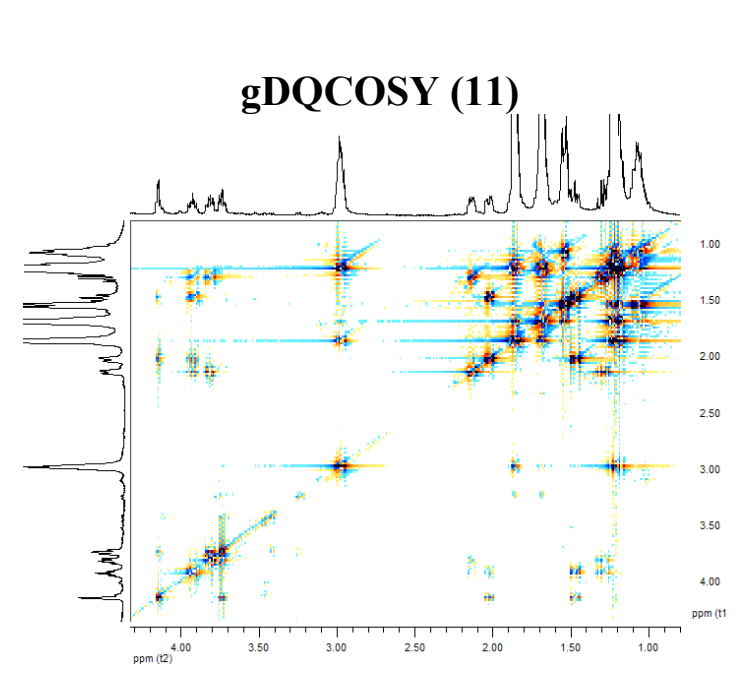

HO'
2-D NMR experiments for 3,5-dideoxy-D-myo-inositol-1phosphate biscyclohexylamine salt (11).

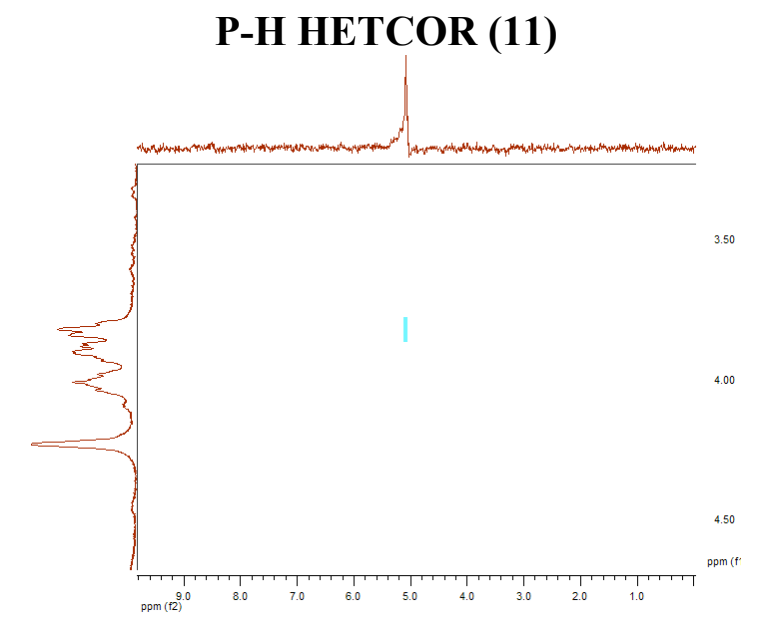


<smiles>O=P(Oc1ccccc1)(Oc1ccccc1)OC1[C@H](OCc2ccccc2)[C@@H](O)[C@@H](OC(=S)Oc2ccccc2)[C@H]1OC(=S)Oc1ccccc1</smiles>

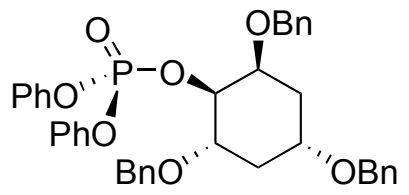<smiles>CO[P+](=O)(O)O[C@H]1[C@@H](O)C[C@@H](O)C[C@H]1O</smiles>

2,4,6-tri-O-benzyl-D-myo-inositol-3-diphenyl phosphate1,5-O-dithiocarbonic acid phenylester (ent-9). Spectral data matches that reported for 9.

2,4,6-tri-O-benzyl-D-myo-inositol-1,5-Dideoxy-3-diphenyl phosphate (ent-10). Spectral data matches that reported for 10. $[\square]_{\mathbf{D}}=+22.4\left(1.0, \mathrm{CH}_{2} \mathrm{Cl}_{2}\right)$.

\section{1,5-Dideoxy-D-myo-inositol-3-phosphate biscyclohexylamine salt (ent-11). Spectral data matches

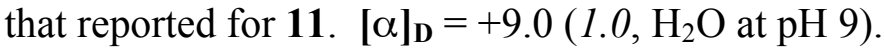

Phosphatase Assays. Phosphatase activity was measured by colorimetric determination of released inorganic phosphate $\left(\mathrm{P}_{\mathrm{i}}\right){ }^{6}$

For A. fulgidus IMPase, the assays were carried out in $50 \mathrm{mM}$ Tris- $\mathrm{HCl}, \mathrm{pH} 8.0,1 \mathrm{mM}$ EDTA and $5 \mathrm{mM} \mathrm{Mg}^{2+}$. The total assay volume is $20 \mathrm{DL}$. The amount of enzyme added was adjusted to give a $15-20 \%$ conversion of substrate to $\mathrm{P}_{\mathrm{i}}$ during the 2 min incubation at $85^{\circ} \mathrm{C}$. For E. coli SuhB, the assay was carried out in the same buffer with $8 \mathrm{mM} \mathrm{Mg}^{2+}$ and no EDTA. The total assay volume is $500 \square \mathrm{L}$, and the assay mixture was incubated at $37{ }^{\circ} \mathrm{C}$ for $1 \mathrm{~min}$. After incubation, $1 \mathrm{~mL}$ of ammonium molybdate and Malachite Green reagent was immediately added to the mixtures. The color reagent contains $\sim 1.2 \mathrm{M} \mathrm{HCl}$ and is sufficient to stop the reaction. A comparison of observed $A_{660}$ changes to those for standard $\mathrm{P}_{\mathrm{i}}$ samples was used to calculate the reaction rate.

${ }^{1}$ Gottlieb, H.E.; Kotlyar, V.; Nudelman, A. J. Org. Chem. 1997, 62, 7512-7515.

${ }^{2}$ Still, W.C.; Kahn, M.; Mitra, J. J. Org. Chem. 1978, 43, 2923.

${ }^{3}$ Sculimbrene, B. R.; Morgan, A. J.; Miller, S. J. J. Am. Chem. Soc. 2002, 124, 11653-11656.

${ }_{5}^{4}$ gDQCOSY: Obtained on Varian Unity INOVA $500 \mathrm{MHz}$ NMR at temp $=28^{\circ} \mathrm{C}$.

${ }^{5}$ P-H HETCOR: Obtained on Varian Unity INOVA $500 \mathrm{MHz}$ NMR at temp $=28{ }^{\circ} \mathrm{C}$. $\mathrm{P}-\mathrm{H}$ coupling constant $(8.2 \mathrm{~Hz})$ determined from ${ }^{31} \mathrm{P}$ NMR without proton decoupling.

${ }^{6}$ Itaya, K.; Ui, M. Clin. Chim. Acta 1966, 14, 361-366. 